\title{
Celestial mechanics of elastic bodies
}

\author{
Robert Beig • Bernd G. Schmidt
}

Published online: 20 June 2009

(C) Springer-Verlag 2009

\section{Erratum to: Math. Z. (2008) 258:381-394 DOI 10.1007/s00209-007-0177-5}

1. In p. 382, after Eq. (2), the function $\hat{w}$ should depend on $(X, \partial \Phi)$, respectively $\left(X, H^{B C}\right)$ rather than $(\Phi, \partial \Phi)$ resp. $\left(\Phi, H^{B C}\right)$.

2. In p. 383, paragraph between Eqs. (5) and (6): the sentence "Homogeneity means that $\hat{w}$ is independent of $\Phi$ " should read "Homogeneity means that $\hat{w}$ is independent of $X$ ".

3. In p. 383, Eq. (7), after Eq. (8) and in Property 1: the first argument of $\hat{\sigma}_{i}^{A}$ should again be $X$.

These errors do not affect the rest of the paper.

The online version of the original article can be found under doi:10.1007/s00209-007-0177-5.

R. Beig $(\bowtie)$

Boltzmanngasse 5, 1090 Vienna, Austria

e-mail: robert.beig@univie.ac.at

B. G. Schmidt

Max-Planck-Institut für Gravitationsphysik Albert-Einstein-Institut,

Am Mühlenberg 1, 14476 Golm, Germany 